\title{
Cory COVID-Bot: An Evidence-Based Behavior Change Chatbot for COVID-19
}

\author{
Simon T. VAN BAAL ${ }^{\mathrm{a}}$, Suong LE ${ }^{\mathrm{b}, \mathrm{c}}$, Farhad FATEHI ${ }^{\mathrm{d}, 1}$, Jakob HOHWY ${ }^{\mathrm{a}, \mathrm{e}}$ and \\ Antonio VERDEJO-GARCIA ${ }^{\mathrm{d}, \mathrm{f}}$ \\ ${ }^{a}$ Cognition and Philosophy Lab, Monash University, Australia \\ ${ }^{b}$ Department of Gastroenterology and Hepatology, Monash Health, Australia \\ ${ }^{c}$ School of Clinical Sciences, Monash University, Australia \\ ${ }^{d}$ School of Psychological Sciences, Monash University, Australia \\ ${ }^{e}$ Centre for Consciousness \& Contemplative Studies, Monash University, Australia \\ ${ }^{f}$ Turner Institute for Brain and Mental Health, Monash University, Australia
}

\begin{abstract}
Cory COVID-Bot is an artificial intelligence chatbot designed and built by a multisector collaboration to help people safely step towards COVID normal. Achieving COVID normal and avoiding unnecessary adverse health outcomes requires effective communication to the public regarding COVID safe behaviors, but reaching young, culturally and linguistically diverse members of the community is challenging for government. Cory COVID-Bot was developed to directly engage with difficult to reach populations in English and Vietnamese. In order to resolve public ambiguity and uncertainty about public health guidelines, and to stimulate safe behavior, Cory COVID-Bot provides updated recommendations and behavior change interventions, which emphasize the importance of COVID safe behaviors.
\end{abstract}

Keywords. Chatbot, COVID-19, Behavior Change, Artificial Intelligence

\section{Introduction}

Promoting adherence to behavioral changes remains a cornerstone strategy to prevent and contain COVID-19 resurgences. Based on the 2009 H1N1 experience, repeated messaging from government and public health experts often does not impact the likelihood of people routinely engaging in infection prevention behaviors [1]. In particular, there has been a global lack of adherence to COVID-safe behaviors in difficult to reach communities due to age, language or ethnicity during the COVID-19 lockdowns.

There is no scalable solution that can simultaneously educate hard-to-reach members of the community and shift human behavior at a general population level. Chatbots are gaining momentum within mobile health settings for lifestyle risk factor modification and chronic disease management. We proposed to overcome communication problems and bridge the COVID-safe "knowledge-action" gap through the rapid development and validation of a user-friendly, low-cost and scalable chatbot, Cory COVID-Bot.

\footnotetext{
${ }^{1}$ Corresponding Author, Farhad Fatehi MD PhD, School of Psychological Sciences, Monash University, 18 Innovation Walk, Clayton VIC 3800, Australia; E-mail: farhad.fatehi@monash.edu.
} 


\section{Methods}

We adopted an agile design approach to build a de novo chatbot as a targeted behavior change intervention tool to combat non-adherence to COVID-19 safe behaviors. The chatbot provides essential information about the prevention, diagnosis and treatment of COVID-19 based on natural language processing that uses training phrases and followup questions in order to engage users in a conversation rather than didactic education. Users can interact with Cory through free text, and he can reply through pre-set responses, media, and resource-carousels. Cory COVID-Bot delivers evidence-based behavioral interventions in the form of short animations. The avatar of the chatbot, Cory, is a community librarian in his mid-thirties. He was designed to be a collaborative model citizen (he can demonstrate how he uses a mask in a social situation); resourceful and pragmatic (he can geolocate the closest testing center); informative and accurate (realtime information regarding the latest restrictions relevant to your area) and empathetic (helps to self-manage loneliness, anxiety, and low mood). Cory COVID-Bot has an affable, knowledgeable, and conscientious persona, able to respectfully interact with all demographics, while providing clear, practical, and actionable information (Figure 1).
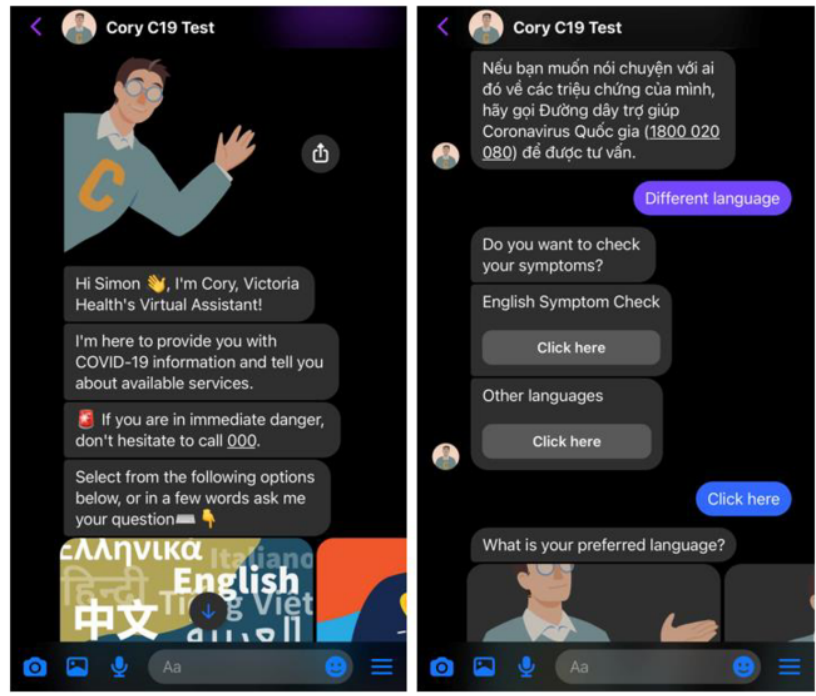

Figure 1. Two screenshots of Cory COVID-Bot deployed in Facebook Messenger app.

The project team developed a series of behavioral interventions to be delivered by Cory for promoting specific actions vital to curtailment of COVID-19. These behavioral interventions were intended to remove mental and practical hurdles to desired COVID safe practices. This is effective because people have an intrinsic tendency to procrastinate decisions if they perceive the decisions as complex, if the consequences seem distant (e.g. present bias), or if they perceive the decision as important $[2,3]$.

The interventions were informed by the literature on behavior change; we focused on two issues with relevance in the pandemic. Altruism (which includes COVID-safe behavior) can be motivated by compassion, which can be trained [4], and that people tend to underestimate exponential disease transmission [5]. The first behavioral 
intervention was aimed at diminishing psychological threshold for COVID-19 testing by providing a clear and resonant rationale for why getting tested is essential for helping others including loved ones. The animation shows a family separated when one of the family members contracts COVID-19. This intervention invoked empathy and compassion in the viewer, which has been shown to increase willingness to comply with public health guidelines [6]. The second behavioral intervention addressed exponential growth bias, in this case the tendency to underestimate the increase of case numbers over time, in order to increase the likelihood of getting tested, address personal responsibility and emphasize the exponential nature of viral transmission [5].

\section{Results}

We built a scalable and practical artificial intelligence (AI) chatbot that can be deployed on various platforms, such as Facebook Messenger, and can therefore be adapted for different purposes as the COVID pandemic evolves. Cory is an AI chatbot capable of delivering targeted behavioral interventions when deemed appropriate in a conversation, thereby increasing its impact as a population-based intervention. In this case, when the individual interacting with Cory asks about COVID-19 symptoms, he would provide them with the necessary information, and prompt them with an animation aimed at eliciting empathy and compassion (see Figure 2), an animation that aims to correct exponential growth bias (see Figure 3), or skips the animations with equal chance.
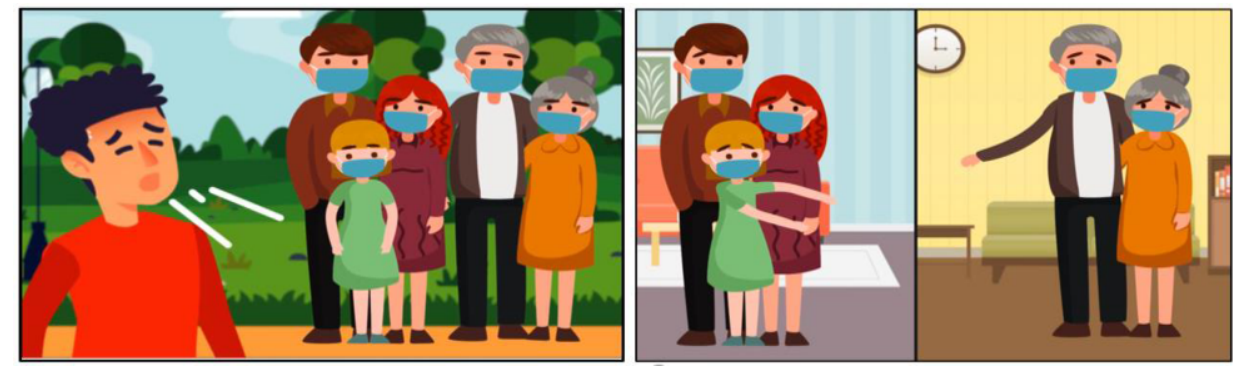

Figure 2. Empathy manipulation, a man did not wear a mask (left), and caused family separation (right).
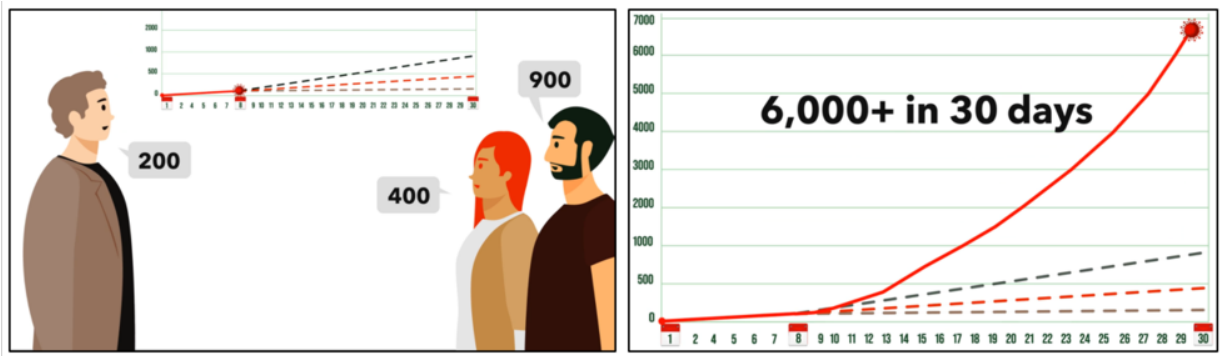

Figure 3. Exponential growth bias intervention, people in the picture on the left guess the trajectory of an outbreak, and an exponential growth curve is traced on the right.

Afterwards, Cory would ask how likely it was the user would get tested. This type of question generates tangible behavior change data, and enables researchers and 
developers to analyze whether the intervention is likely to be effective. We thus prepared Cory for conducting a randomized controlled trial, the results of which will be communicated after testing is completed. This setup also allows for continual updating and fine-tuning of the behavioral interventions.

\section{Discussion}

This project shows that it is possible to develop an artificial intelligence chatbot that informs people about public health guidelines and delivers targeted behavioral interventions. Cory is low-cost, adaptable and agile. Once Cory has engaged with a user, he can maintain a direct line of communication through push notifications. The design allows for translation into different languages, swift adjustments in light of new information, and adaptation to new use potential such as addressing vaccine hesitancy. These are all desirable attributes in a pandemic where information readily changes, misinformation and disinformation are rife on social media channels, and at-risk communities need to selectively target.

We have found that behavioral interventions delivered via animations allow for more degrees of freedom than text-based interventions, which means more effective manipulations can be pursued through iteration. Various strategies can be used to identify whether the chatbot has been effective at achieving the aims set by the project team, which the team can use to update the chatbot content and functionality. Chatbots could play a meaningful role in the pandemic, and could be considered for deployment on platforms that are popular among hard-to-reach communities as both a trusted advisor and an unbiased source of information.

\section{References}

[1] Gray L, MacDonald C, Mackie B, Paton D, Johnston D, Baker MG. Community responses to communication campaigns for influenza A (H1N1): a focus group study. BMC Public Health. 2012 Dec;12(1):1-2.

[2] Beshears J, Choi JJ, Laibson D, Madrian BC. The importance of default options for retirement saving outcomes: Evidence from the United States. Social security policy in a changing environment: University of Chicago Press; 2009. p. 167-95.

[3] O'Donoghue T, Rabin M. Choice and procrastination. The Quarterly Journal of Economics. 2001;116(1):121-60.

[4] Weng, HY, Fox, AS, Shackman, AJ, Stodola, DE, Caldwell, JZ, Olson, MC, Rogers, GM, Davidson, RJ. Compassion training alters altruism and neural responses to suffering. Psychological science, 2013;24(7):1171-1180.

[5] Lunn PD, Timmons S, Belton CA, Barjaková M, Julienne H, Lavin C. Motivating social distancing during the Covid-19 pandemic: An online experiment. Social Science \& Medicine. 2020;265:113478.

[6] Pfattheicher S, Nockur L, Böhm R, Sassenrath C, Petersen MB. The Emotional Path to Action: Empathy Promotes Physical Distancing and Wearing of Face Masks During the COVID-19 Pandemic. Psychological Science. 2020;31(11):1363-73. 